\title{
Network Structures with Hierarchy and Communication
}

\author{
Encarnación Algaba ${ }^{1} \cdot$ René van den Brink $^{2}$ (D) Chris Dietz ${ }^{2}$
}

Received: 24 July 2017 / Accepted: 6 July 2018 / Published online: 26 July 2018

(c) The Author(s) 2018

\begin{abstract}
Agents, participating in different kind of organizations, usually take different positions in some network structure. Two well-known network structures are hierarchies and communication networks. This paper aims at introducing a new type of network structure having both communication and hierarchical features. We describe a network by a collection of feasible sets, being the sets of network positions (nodes), that can organize themselves and act as a group. We introduce a new type of network structure, that has communication as well as hierarchical features. We compare these new network structures with other structures from the literature, and study its basis, i.e. the 'smallest' representation of the network. Finally, we apply these new network structures to cooperative games, where cooperation is restricted by some network structure, and provide an axiomatization of an extension of the Shapley value to this class of games.
\end{abstract}

Keywords Game theory · Cooperative TU-game · Networks · Communication · Hierarchy

Mathematics Subject Classification 91A12 · 91A43

$\varangle$ René van den Brink

jrbrink@feweb.vu.nl

Encarnación Algaba

ealgaba@us.es

Chris Dietz

cdietz@feweb.vu.nl

1 Department of Applied Mathematics II and IMUS, Escuela Superior de Ingenieros,

Camino de los Descubrimientos, s/n, 41092 Sevilla, Spain

2 Department of Econometrics and Tinbergen Institute, VU University, De Boelelaan 1105,

1081 HV Amsterdam, The Netherlands 


\section{Introduction}

The most common types of network structures, that are applied in the literature, are represented by an undirected or directed graph. However, many real applications cannot be described by such graphs. Although they can be described by the more general hypergraphs, this last class is so general, that it does not capture some essential features of certain network structures. The goal of the underlying paper is to get a better understanding of organizations, that have hierarchical as well as communication features.

In the field of restricted cooperation in cooperative games, communication restrictions are usually modelled by an undirected graph, whose set of nodes are the positions of the players in some communication network, while the edges or links of the graph represent bilateral communication links. A set of players (called a coalition) can only cooperate, if the players in the coalition are connected in the network in such a way that there is a path between each two players in the coalition, that uses only players from the coalition; see Myerson [1]. This model is generalized by Algaba et al. $[2,3]$, where the players in the game are part of a network structure, that is modelled as a union stable network structure, being a set of (feasible) coalitions such that the union of every pair of non-disjoint coalitions is also feasible; see also Algaba et al. $[4,5]$. Here, for two non-disjoint coalitions, i.e. coalitions with a nonempty intersection, the players that belong to both coalitions act as intermediaries, allowing the two coalitions to cooperate together. This property is obviously satisfied by the set of connected coalitions of any undirected graph, ${ }^{1}$ see also the special case of partition systems [7].

A model that studies restrictions in cooperation arising from hierarchies is that of a game with a permission structure developed in Gilles et al. [8], Gilles and Owen [9], van den Brink and Gilles [10] and van den Brink [11]. In this model, it is assumed that the players are part of a hierarchical organization, which is represented by a directed graph, ${ }^{2}$ where some players need permission or approval from their superior players before they are allowed to cooperate. In Algaba et al. [14], this is generalized by showing that the sets of feasible coalitions, arising from these permission structures, are antimatroids, being well-known combinatorial structures, representing hierarchies, see Dilworth [15] and Edelman and Jamison [16]. A set of feasible coalitions is an antimatroid, if (i) it contains the empty set, (ii) satisfies accessibility (meaning that every nonempty feasible coalition has at least one player such that without this player the remaining coalition is still feasible), and (iii) is union closed (meaning that the union of any two feasible coalitions is also feasible).

In van den Brink [17], a distinction is made between hierarchies and communication networks by showing that the sets of coalitions, that can be the set of communication feasible sets (sets of coalitions that can be obtained as the set of connected coalitions in some undirected graph), are exactly those sets of coalitions that, besides containing the empty set, satisfy the above-mentioned union stability and 2-accessibility (meaning

1 Union stable network structures are closely related to hypergraphs, see Algaba et al. [6].

2 Another model dealing with hierarchies, defined by digraphs, is that of games under predecence constraints, introduced by Faigle and Kern [12], and recently studied in Algaba et al. [13]. 
Table 1 Comparing properties of communication feasible sets and antimatroids

\begin{tabular}{lll}
\hline Communication feasible sets & & Antimatroids \\
\hline Feasible empty set & & Feasible empty set \\
Union stable & $\Leftarrow$ & Union closed \\
2-Accessible & $\Rightarrow$ & Accessible \\
\hline
\end{tabular}

that every feasible coalition with two or more players has at least two players, that can leave the coalition such that the remaining sets of players are still feasible coalitions). So, compared to communication feasible sets, antimatroids satisfy a stronger union property (since union closedness implies union stability), but a weaker accessibility property (since 2 -accessibility implies accessibility), see Table 1.

Considering union stability as a property of a network structure that reflects communication, and accessibility as a property that reflects hierarchy, network structures that satisfy these two properties seem the most specific type of network structure, that combines hierarchy with communication. Therefore, we introduce accessible union stable network structures, where accessibility reflects the hierarchy and union stability reflects the communication feature of the network. Obviously, this type of network structure generalizes the connected coalitions in an undirected graph, as well as antimatroids.

Although union stable network structures are even more general, a main advantage of the new network structures, introduced in this paper, is that they also take into account the hierarchical features in the network, and not only the communication characteristics. We compare the new network structures with known network structures from the literature. In particular, we show that the class of accessible union stable network structures contains the class of so-called augmenting systems as a proper subclass. Augmenting systems are introduced in Bilbao [18] and, compared to accessible union stable network structures, instead of accessibility they satisfy an augmentation property, saying that whenever a feasible coalition is a subset of another feasible coalition, there is always at least one player in the larger coalition that can be added to the smaller coalition. This gives the interesting insight that, under union stability (that is, communication), being able to let feasible coalitions grow by adding players one by one implies that we can shrink every feasible coalition by deleting players one by one, but not the other way around. This has important consequences, for example, when we apply network structures in the coalition formation process in cooperative games.

Finally, we apply accessible union stable network structures to model cooperation restrictions in cooperative games.

The paper is organized as follows. In Sect. 2, we formally introduce accessible union stable network structures and study some of their properties. In Sect. 3, we consider the basis of these structures. In Sect. 4, we apply these network structures to model cooperation restrictions in cooperative games and provide an axiomatization of a Shapley value for such games. Finally, Sect. 5 contains concluding remarks. 


\section{Accessible Union Stable Network Structures}

For a finite set $N$, a network structure on $N$ is a pair $(N, \mathcal{F})$, where $\mathcal{F} \subseteq 2^{N}$ is a family of subsets of $N$. The sets belonging to $\mathcal{F}$ are called feasible.

By taking the weaker union and accessibility properties, that communication feasible sets and antimatroids have in common (see Table 1), we model organizations, having hierarchical as well as communication features, by those network structures $\mathcal{F} \subseteq 2^{N}$, that contain the empty set and satisfy union stability and accessibility.

Definition 2.1 A network structure $\mathcal{F} \subseteq 2^{N}$ is an accessible union stable network structure, if it satisfies the following properties:

(feasible empty set) $\varnothing \in \mathcal{F}$,

(union stability) $\quad S, T \in \mathcal{F}$ with $S \cap T \neq \emptyset$, implies that $S \cup T \in \mathcal{F}$,

(accessibility) $\quad S \in \mathcal{F}$ with $S \neq \emptyset$, implies that there exists $i \in S$, such that $S \backslash\{i\} \in \mathcal{F}$.

We say that a network structure $\mathcal{F} \subseteq 2^{N}$ is normal, if for every $i \in N$ there is a feasible coalition $S \in \mathcal{F}$, such that $i \in S$.

Obviously, antimatroids and communication feasible sets are accessible union stable network structures. Other obvious examples on $N=\{1, \ldots, n\}$ are $\mathcal{F}=2^{N}$, $\mathcal{F}=\{\emptyset,\{i\}\}$ for $i \in N$, and $\mathcal{F}=\{\emptyset,\{1\}, \ldots,\{n\}\}$.

Union stability has a natural and intuitive interpretation in the context of partial cooperation, since it expresses that players, who belong to the intersection of two feasible coalitions, can act as intermediaries and generate cooperation within the union of the two coalitions. However, when only requiring union stability, it is not clear what is the hierarchical feature of an organization. In the new structures, the hierarchy is reflected by accessibility.

An already existing network structure, that is comparable with the new structures, also containing all communication feasible sets and antimatroids, are augmenting systems [18].

Definition 2.2 A network structure $\mathcal{F} \subseteq 2^{N}$ is an augmenting system, if it satisfies the following properties:

(feasible empty set) $\varnothing \in \mathcal{F}$,

(union stability) $\quad S, T \in \mathcal{F}$ with $S \cap T \neq \emptyset$, implies that $S \cup T \in \mathcal{F}$,

(augmentation) $\quad S, T \in \mathcal{F}$ with $S \subset T$, implies that there exists $i \in T \backslash S$, such that $S \cup\{i\} \in \mathcal{F}$.

Augmentation establishes that, whenever there are two feasible coalitions, such that one is contained in the other, we can keep adding players from the 'bigger' coalition to the 'smaller' coalition one by one, such that after each addition the new coalition is feasible.

Let $\mathcal{F} \subseteq 2^{N}$ be an accessible union stable network structure. A player $i \in S, S \in \mathcal{F}$, such that $S \backslash\{i\} \in \mathcal{F}$ is called an extreme player of coalition $S$ in $\mathcal{F}$, i.e. an extreme player is a player, who can be deleted from a feasible coalition keeping feasibility. A player $i \in N \backslash S$, with $S \in \mathcal{F}$, such that $S \cup\{i\} \in \mathcal{F}$, is called an augmenting player of 
coalition $S$ in $\mathcal{F}$, i.e. an augmenting player is a player who can be joined to a feasible coalition keeping feasibility.

In fact, the augmentation property implies accessibility. Indeed, for $T \in \mathcal{F}$, with $T \neq \emptyset$ and $t=|T|$, by the augmentation property, there exists a sequence of coalitions $\left(T_{0}, T_{1}, \ldots, T_{t}\right)$, with $T_{h} \in \mathcal{F},\left|T_{h}\right|=h, 0 \leq h \leq t$, such that $\emptyset=T_{0} \subset T_{1} \subset \cdots \subset$ $T_{t-1} \subset T_{t}=T$. Therefore, there exists a player $i \in T$, such that $T \backslash\{i\}=T_{t-1} \in$ $\mathcal{F}$. This shows that augmenting systems satisfy accessibility, and therefore, they are accessible union stable network structures.

Proposition 2.1 If $\mathcal{F} \subseteq 2^{N}$ is an augmenting system, then $\mathcal{F}$ is an accessible union stable network structure.

However, accessibility does not imply augmentation, i.e. there are accessible union stable network structures, that are not augmenting systems. Next, we discuss an application of accessible union stable network structures, that is neither an augmenting system, antimatroid or a communication feasible set.

Example 2.1 (Exploring and careful societies) (Fig. 1) Consider two societies, that each live in their own country. Within each society, there are no restrictions in cooperation, and all subsets of the society members are feasible. But one of the two societies is very careful and the members of that society do not dare to cross the border to leave their own country. The agents in the other society are explorers, and each (or any subset) of them dares to cross the border to the 'careful' society, and can meet there the society of all careful players as a whole. Let $N$ and $M$ be the two societies with $N \cap M=\emptyset$, where $N$ represents the society of 'explorers' and $M$ the society of 'careful' players. So, besides every subset of $N$ and of $M$, every subset of society $N$ can form a feasible coalition with all players in $M$. The players in $M$ can only cooperate with players from $N$, if all players from $M$ join together. If $N=\{1,2\}$ is the society of explorers, and $M=\{3,4,5\}$ is the society of careful players, then the resulting feasible sets are those in

$$
\begin{aligned}
\mathcal{F}= & \{\emptyset,\{1\},\{2\},\{1,2\},\{3\},\{4\},\{5\},\{3,4\},\{3,5\},\{4,5\},\{3,4,5\}, \\
& \{1,3,4,5\},\{2,3,4,5\},\{1,2,3,4,5\}\}
\end{aligned}
$$

(where the first row of $\mathcal{F}$ contains $N, M$ and all their subsets, while in the second row are the feasible sets that contain players from both societies).

The network structure $\mathcal{F}$, given by (1), is union stable since (i) any union of two coalitions, that are either a subset of $N$ or a subset of $M$, is feasible, and (ii) the union of two non-disjoint feasible coalitions, containing players from $N$ and $M$, must contain all players from $M$ (since at least one of these two coalitions must contain players from $N$ and $M$, and thus, it must contain all players from $M$ ), and therefore, it is feasible.

The network structure is accessible since (i) for each nonempty feasible coalition, that is a subset of $N$ or $M$, every player in this coalition can be deleted keeping feasibility, and (ii) for every feasible coalition that contains players from both $N$ and $M$, each player from $N$ can be deleted. The resulting coalition still contains all players from $M$, and then, it is feasible. 
Fig. 1 Accessible union stable network structure $(N, \mathcal{F})$

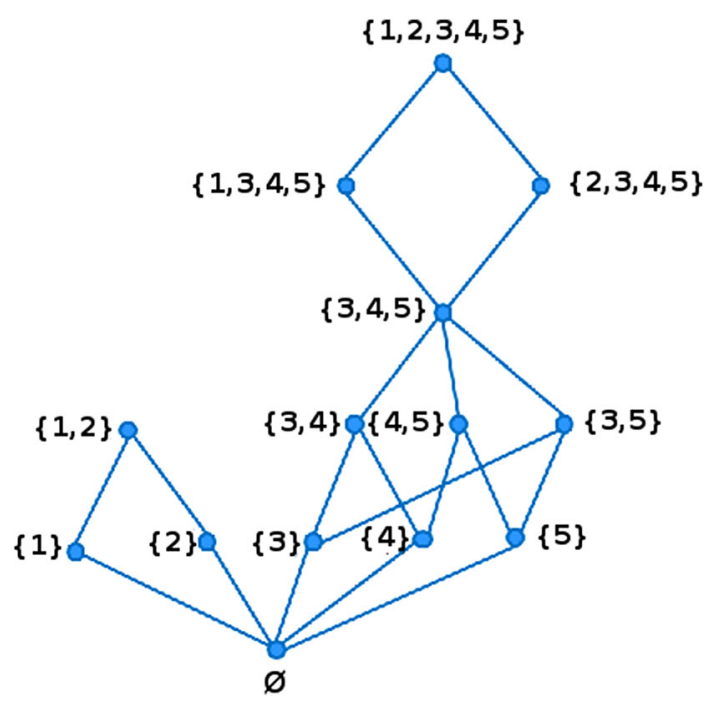

To show that the network structure $\mathcal{F}$ is not a communication feasible set, consider, for example, feasible coalition $\{2,3,4,5\}$. This coalition contains one player from $N$, player 2, and all players from $M$. But since none of the players of $M$ can be deleted, player 2 is the only extreme player, so the network structure does not satisfy 2-accessibility.

To show that the network structure $\mathcal{F}$ is not an antimatroid, consider, for example, coalitions $\{1\}$ and $\{3\}$. These are proper subsets of $N$, respectively $M$, so their union $\{1,3\}$ contains a player from $N$ and a player from $M$, but it does not contain all players from $M$, and therefore, it is not feasible, showing that $\mathcal{F}$ does not satisfy union closedness.

Finally, to show that the network structure $\mathcal{F}$ is not an augmenting system, consider coalitions $N=\{1,2\}$ and $N \cup M=\{1,2,3,4,5\}$. Then, no single player from $M$ can be added to $N$ to get a feasible coalition, since the players of $M$ only join $N$ as a group, showing that $\mathcal{F}$ does not satisfy augmentation.

An interesting relation between accessible union stable network structures and antimatroids is that every network structure, such that the collection of feasible coalitions containing a particular player is an antimatroid, is an accessible union stable network structure.

Proposition 2.2 Let $\mathcal{F} \subseteq 2^{N}$ be a network structure containing the empty set. If $\mathcal{F}_{i}=\{\emptyset\} \cup\{T \in \mathcal{F}: i \in T\}$ is an antimatroid for all $i \in N$, then $\mathcal{F}$ is an accessible union stable network structure.

Proof Consider $S, T \in \mathcal{F}$ with $S \cap T \neq \emptyset$. If $j \in S \cap T$ then $S, T \in \mathcal{F}_{j}$. Since $\mathcal{F}_{j}$ is union closed it holds that $S \cup T \in \mathcal{F}_{j}$, and hence $S \cup T \in \mathcal{F}$, showing union stability of $\mathcal{F}$.

To show accessibility of $\mathcal{F}$, consider $S \in \mathcal{F}, S \neq \emptyset$. Then, $S \in \mathcal{F}_{j}$ for some $j \in S$. But then, there exists an $h \in S$ such that $S \backslash\{h\} \in \mathcal{F}_{j}$ by accessibility of $\mathcal{F}_{j}$. Hence, $S \backslash\{h\} \in \mathcal{F}$, showing accessibility of $\mathcal{F}$. 
Fig. 2 Accessible union stable network structure $(N, \mathcal{F})$ of Example 2.2

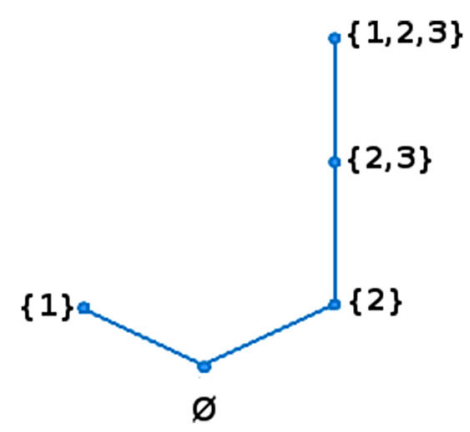

The reverse is not true as shown in the following example.

Example 2.2 Consider the network structure $\mathcal{F}=\{\emptyset,\{1\},\{2\},\{2,3\}, N\}$ on $N=$ $\{1,2,3\}$. This is an accessible union stable network structure (Fig. 2), but the set system $\mathcal{F}_{1}=\{\emptyset,\{1\}, N\}$ is not an antimatroid, since it does not satisfy accessibility (no single player can be deleted from $N$ ).

The dual structure $\mathcal{F}^{d}$ of network structure $\mathcal{F} \subseteq 2^{N}$ consists of all coalitions whose complement is feasible, i.e. $\mathcal{F}^{d}=\{S \subseteq N: N \backslash S \in \mathcal{F}\}$.

Note that a coalition may belong to an accessible union stable network structure as well as to its dual structure. It is known that convex geometries, being a combinatorial abstraction of convex sets introduced by Edelman and Jamison [16], are the dual structures of antimatroids. A convex geometry is a network structure, that contains the empty set, is intersection closed and satisfies an alternative augmentation property saying that for every feasible coalition, that is a proper subset of the 'grand coalition' $N$, there is a player that can be added resulting in another feasible coalition with one more player. ${ }^{3}$ Note that the alternative augmentation property implies that the 'grand coalition' is feasible, and thus convex geometries are normal network structures.

The dual structures of accessible union stable network structures form a class of network structures that contain all convex geometries.

Definition 2.3 A network structure $\mathcal{F} \subseteq 2^{N}$ is an intersecting stable network structure with the augmentation property, if it satisfies the following properties:

(feasible empty set) $\quad \emptyset \in \mathcal{F}$,

(weak intersection closed) $S, T \in \mathcal{F}$ with $S \cup T \neq N$, implies that $S \cap T \in \mathcal{F}$

(augmentation 2) $\quad S \in \mathcal{F}$ with $S \neq N$, implies that there exists $i \in N \backslash S$ such that $S \cup\{i\} \in \mathcal{F}$.

Proposition 2.3 Let $\mathcal{F} \subseteq 2^{N}$ be a network structure with $N \in \mathcal{F}$. Then, $\mathcal{F}$ is an accessible union stable network structure if and only if $\mathcal{F}^{d}$ is an intersecting stable network structure with the augmentation property.

Proof The sets $\emptyset$ and $N$ belong to $\mathcal{F}$, and thus also to $\mathcal{F}^{d}$. Note that

\footnotetext{
3 Edelman and Jamison [16] showed that $L$ is a connected block graph, if and only if the collection of subsets of $N$, which induces connected subgraphs, is a convex geometry.
} 
Fig. 3 Intersecting stable network structure $(N, \mathcal{F})$ of Example 2.3

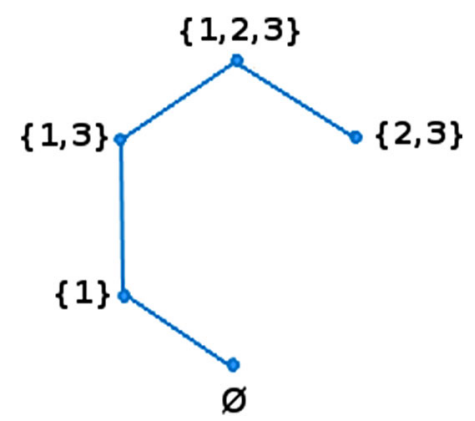

(i) $S, T \in \mathcal{F} \Leftrightarrow(N \backslash S),(N \backslash T) \in \mathcal{F}^{d}$,

(ii) $S \cap T \neq \emptyset \Leftrightarrow(N \backslash S) \cup(N \backslash T) \neq N$, and

(iii) $S \cup T \in \mathcal{F} \Leftrightarrow N \backslash(S \cup T)=(N \backslash S) \cap(N \backslash T) \in \mathcal{F}^{d}$.

Therefore, union stability of $\mathcal{F}$ implies that $\mathcal{F}^{d}$ is weak intersection closed. Finally, for any $S \subseteq N$ with $i \in S$, it holds $N \backslash S \neq N, i \in N \backslash(N \backslash S)=S$ and,

(iv) $S, S \backslash\{i\} \in \mathcal{F} \Leftrightarrow N \backslash S \in \mathcal{F}^{d}, N \backslash(S \backslash\{i\})=(N \backslash S) \cup\{i\} \in \mathcal{F}^{d}$.

Therefore, accessibility of $\mathcal{F}$ implies that $\mathcal{F}^{d}$ satisfies augmentation 2.

Given an intersecting stable network structure with the augmentation property $\mathcal{F} \subseteq$ $2^{N}$, by augmentation 2 , every $S \in \mathcal{F}, S \neq N$, has at least one augmenting player. However, the set of extreme players of some coalitions can be empty.

Example 2.3 Consider the network structure $\mathcal{F}=\{\emptyset,\{1\},\{1,3\},\{2,3\}, N\}$ on $N=$ $\{1,2,3\}$. This is an intersecting stable network structure with the augmentation property with $\operatorname{ex}(\{2,3\})=\emptyset($ Fig. 3$)$.

Note that, if an intersecting stable network structure with the augmentation property is an antimatroid, then it is closed under union. The reverse is not true, as also follows from the intersecting stable network structure of Example 2.3. This example is union closed but is not an antimatroid, since coalition $\{2,3\}$ has no extreme players.

\section{The Supports of an Accessible Union Stable Network Structure}

In many cases, it is more transparent to represent a network structure by its supports, being a subset of the network structure from which, with some specified operations, we can generate the full network structure. For example, if we know that we are dealing with communication in an undirected graph, then we usually do not describe it as all connected coalitions, but just the edges being the feasible coalitions of size two. We can generate all connected coalitions by applying union stability, as done below for any accessible union stable network structure. In fact, the edges and all singletons form the supports of an undirected communication graph.

Let $\mathcal{F}$ be a union stable network structure on $N$, and consider the set

$R(\mathcal{F})=\{R \in \mathcal{F}:$ there are $S, T \in \mathcal{F}, S \neq R, T \neq R, S \cap T \neq \emptyset$ with $R=S \cup T\}$ 
Fig. 4 Accessible union stable network structure $(N, \mathcal{F})$ of Example 3.1

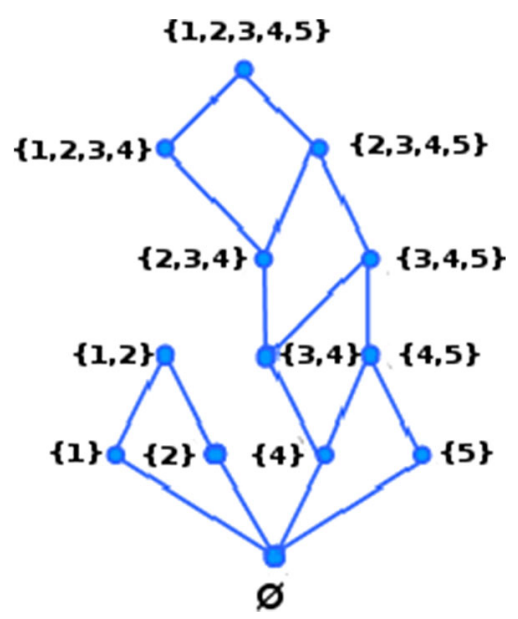

consisting of those feasible coalitions, which can be written as the union of two distinct feasible coalitions with a nonempty intersection. The other feasible coalitions (i.e. those that cannot be written as the union of two other feasible coalitions with a nonempty intersection) form the basis of the accessible union stable network structure, in the following sense. For arbitrary union stable network structure $\mathcal{F}$, Algaba et al. [2] inductively define families $\mathcal{G}^{(m)}$, with $m=0,1, \ldots$, and $\mathcal{G}^{(m-1)} \subseteq \mathcal{G}^{(m)} \subseteq \mathcal{F}$, by

$\mathcal{G}^{(0)}=\mathcal{F} \backslash R(\mathcal{F}), \quad \mathcal{G}^{(m)}=\left\{S \cup T: S, T \in \mathcal{G}^{(m-1)}, S \cap T \neq \emptyset\right\}, \quad(m=1,2, \ldots)$.

By union stability, there exists a smallest integer $k$ such that $\mathcal{G}^{(m)}=\mathcal{F}$ for all $m \geq k$. So, every union stable network structure is obtained by starting with the basis, inductively add all unions of non-disjoint feasible coalitions. The set $B(\mathcal{F})=\mathcal{F} \backslash R(\mathcal{F})$ is called the basis of network structure $\mathcal{F}$. The elements of $B(\mathcal{F})$ are called the supports of $\mathcal{F}$.

Example 3.1 Consider the accessible union stable network structure $\mathcal{F}$ on the set players $N=\{1,2,3,4,5\}$, given by (Fig. 4)

$$
\mathcal{F}=\left\{\begin{array}{l}
\emptyset,\{1\},\{2\},\{4\},\{5\},\{1,2\},\{3,4\},\{4,5\},\{2,3,4\}, \\
\{3,4,5\},\{1,2,3,4\},\{2,3,4,5\}, N
\end{array}\right\} .
$$

The supports of $\mathcal{F}$ are given by

$$
B(\mathcal{F})=\{\emptyset,\{1\},\{2\},\{4\},\{5\},\{1,2\},\{3,4\},\{4,5\},\{2,3,4\}\} .
$$

We introduced accessible union stable network structures as a model that generalizes communication feasible sets as well as hierarchies represented by antimatroids in such a way, that two defining properties reflect communication (union stability) and hierarchy (accessibility). Next, we want to see how these features influence the basis and supports of the network structure. The supports of a communication feasible 
Fig. 5 Accessible union stable network structure $(N, \mathcal{F})$ of Example 3.2

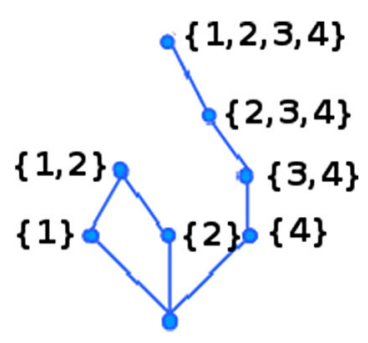

set are exactly those elements that have cardinality one or two, the first type being the singletons and the second type being the links or edges of the communication graph. Given a network structure satisfying 2-accessibility, we can find the edges by repeatedly applying 2 -accessibility to feasible coalitions with more than two elements. The supports of an antimatroid are the paths, being those feasible coalitions, that have exactly one extreme player. Every coalition in an antimatroid is either a path or a union of paths.

Applying accessibility, it holds that $\operatorname{ex}(S) \neq \emptyset$, for any nonempty $S \in \mathcal{F}$, where $\operatorname{ex}(S)$ is the set of extreme players of $S$ in $\mathcal{F}$. For accessible union stable network structures, it turns out that every support either has cardinality at most two (as in communication feasible sets) or is a path (as in antimatroids). In the accessible union stable network structure of Example 3.1, the supports with cardinality at most two are those in $B(\mathcal{F}) \backslash\{\{2,3,4\}\}$, while support $\{2,3,4\}$ is a path with extreme player 2 .

Proposition 3.1 Let $\mathcal{F} \subseteq 2^{N}$ be an accessible union stable network structure. If $B \in$ $B(\mathcal{F})$ with $|B|>2$, then $|\operatorname{ex}(B)|=1$, i.e. $B$ is a path.

Proof Suppose that $B \in B(\mathcal{F})$ is a support of $\mathcal{F}$ such that $|B|>2$ and $\mid$ ex $(B) \mid>1$, i.e. $B$ has at least two extreme players. Then, there exist players $i, j \in B$, with $i \neq j$, such that $B \backslash\{i\} \in \mathcal{F}$ and $B \backslash\{j\} \in \mathcal{F}$. Therefore,

$$
(B \backslash\{i\}) \cup(B \backslash\{j\})=B \text { and }(B \backslash\{i\}) \cap(B \backslash\{j\})=B \backslash\{i, j\} \neq \emptyset \text {, }
$$

which contradicts the fact that $B$ is a support of $\mathcal{F}$.

The reverse is not true.

Example 3.2 Consider the accessible union stable network structure on the set $N=$ $\{1,2,3,4\}$ given by $\mathcal{F}=\{\emptyset,\{1\},\{2\},\{4\},\{1,2\},\{3,4\},\{2,3,4\}, N\}$ (Fig. 5).

Its basis is $B(\mathcal{F})=\{\{1\},\{2\},\{4\},\{1,2\},\{3,4\},\{2,3,4\}\}$. Since the only extreme player of the 'grand coalition' $N$ is player 1 , the grand coalition is a path, but it is not a support since it is the union of $\{1,2\}$ and $\{2,3,4\}$.

It turns out that the basis of an accessible union stable network structure is much more difficult to use than that of the more specific communication feasible sets or antimatroids, but also than the more general one corresponding to union stable structures. The reason is that supports can be 'edges' (coalitions of size two) or paths. For example, in a union stable structure, a coalition is either a support or union of non-disjoint 

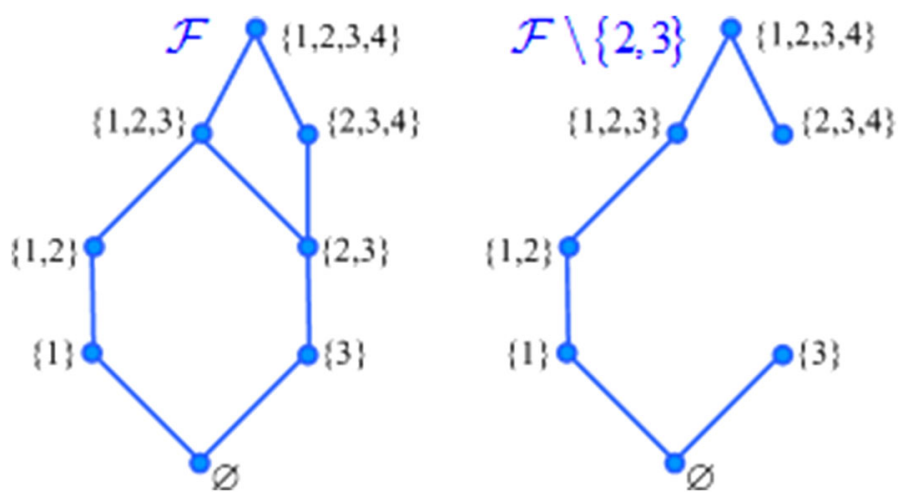

Fig. 6 Network structures $(N, \mathcal{F})$ and $(N, \mathcal{F} \backslash\{2,3\})$ of Example 3.3

supports. This implies that deleting a support from a union stable structure, what is left is still union stable. However, when deleting a support from an accessible union stable network structure, union stability is still satisfied, but the structure need not be accessible anymore.

Example 3.3 Consider the accessible union stable network structure on the set $N=$ $\{1,2,3,4\}$, given by $\mathcal{F}=\{\varnothing,\{1\},\{3\},\{1,2\},\{2,3\},\{1,2,3\},\{2,3,4\}, N\}$.

Its basis is $B(\mathcal{F})=\{\emptyset,\{1\},\{3\},\{1,2\},\{2,3\},\{2,3,4\}\}$.

Deleting the support $\{2,3\}$, the resulting structure is not accessible anymore, since $\{2,3,4\}$ has no extreme player anymore (Fig. 6).

\section{An Application: Cooperative Games on Accessible Union Stable Network Structures}

In this section, we apply accessible union stable network structures to model networks among players in a cooperative TU-game, that have hierarchical as well as communication features. Special cases are the games with restricted communication, and games with hierarchical structures such as games with a permission structure or games on antimatroids.

A situation, in which a finite set of players can obtain certain payoffs by cooperation, can be described by a cooperative game with transferable utility, or simply a TUgame, being a pair $(N, v)$, where $N \subseteq \mathbb{N}$ is a finite set of players and $v: 2^{N} \rightarrow \mathbb{R}$ is a characteristic function satisfying $v(\emptyset)=0$. For any coalition $S \subseteq N, v(S)$ is the worth of coalition $S$, being the total payoff that the members of coalition $S$ can obtain by cooperation. Since we take the player set to be fixed, we denote a TU-game $(N, v)$ just by its characteristic function $v$. We denote the collection of all TU-games on player set $N$ by $\mathcal{G}^{N}$.

A payoff vector of an $n$-player TU-game $v \in \mathcal{G}^{N}$ is an $n$-dimensional vector $x \in \mathbb{R}^{N}$, giving a payoff $x_{i} \in \mathbb{R}$ to any player $i \in N$. A (single-valued) solution for TU-games is a mapping $f$, that assigns to every game $v \in \mathcal{G}^{N}$ a payoff vector 
$f(v) \in \mathbb{R}^{N}$. One of the most well-known solutions for TU-games is the Shapley value (Shapley [19]) given by

$$
S h_{i}(v)=\sum_{\substack{S \subseteq N \\ i \in S}} \frac{(|N|-|S|) !(|S|-1) !}{|N| !}(v(S)-v(S \backslash\{i\})) \quad \text { for all } i \in N,
$$

where $|S|$ denotes the cardinality of (number of elements in) $S \subseteq N$. In a TU-game, any subset $S \subseteq N$ is assumed to be able to form a coalition and earn the worth $v(S)$. However, in most economic, political and OR applications not every set of participants can form a feasible coalition. Therefore, cooperative game theory models have been developed, that take restrictions on coalition formation into account. This can be modelled by considering a set of feasible coalitions $\mathcal{F} \subseteq 2^{N}$, that need not contain all subsets of the player set $N$.

We assume that $\mathcal{F} \subseteq 2^{N}$ is an accessible union stable network structure. For $S \subseteq N$, the maximal nonempty feasible subsets of $S$ are called components of $S$, i.e. a coalition $R \in \mathcal{F}$ is a component of $S$ in $\mathcal{F}$, if there is no $T \in \mathcal{F}$, with $R \subset T \subseteq S$. We denote the set of the components of $S \subseteq N$ by $C_{\mathcal{F}}(S)$. Observe that $C_{\mathcal{F}}(S)$ may be the empty set. As shown in Algaba et al. [2], a network structure $\mathcal{F} \subseteq 2^{N}$ is union stable if and only if for any $S \subseteq N$, with $C_{\mathcal{F}}(S) \neq \emptyset$, the components of $S$ form a partition of a subset of $S$.

A game on an accessible union stable network structure is a triple $(N, v, \mathcal{F})$, where $v: 2^{N} \rightarrow \mathbb{R}$, with $v(\emptyset)=0$, is a characteristic function, and $\mathcal{F} \subseteq 2^{N}$ is an accessible union stable network structure. Since, again we take the player set to be fixed, we denote a game on an accessible union stable network structure $(N, v, \mathcal{F})$ by $(v, \mathcal{F})$. The set of all games on an accessible union stable network structure with player set $N$ is denoted by $G A U S^{N}$.

Definition 4.1 Let $v: 2^{N} \rightarrow \mathbb{R}$ be a cooperative game, and let $\mathcal{F} \subseteq 2^{N}$ be an accessible union stable network structure. The restricted game $v^{\mathcal{F}}: 2^{\bar{N}} \rightarrow \mathbb{R}$, is defined by

$$
v^{\mathcal{F}}(S)=\sum_{T \in C_{\mathcal{F}}(S)} v(T) \text { for all } S \subseteq N
$$

Notice that $v^{\mathcal{F}}(S)=0$, if $C_{\mathcal{F}}(S)=\emptyset$. If $\mathcal{F}$ is the accessible union stable network structure, given by the connected coalitions in a communication graph $(N, L)$, then $v^{\mathcal{F}}$ is the graph-restricted game of Myerson [1].

Example 4.1 Consider game $v$, given by $v(S)=1$, if $\{1,5\} \subseteq S$, and $v(S)=0$, otherwise, and the communication graph $L=\{\{1,2\},\{1,3\},\{2,4\},\{3,4\},\{4,5\}\}$. Taking $\mathcal{F}=\mathcal{F}_{\mathcal{L}}$ as the set of connected coalitions in graph $L$, the restricted game is given by $v^{\mathcal{F}_{\mathcal{L}}}(S)=1$, if $S \in\{\{1,2,4,5\},\{1,3,4,5\},\{1,2,3,4,5\}\}$, and $v(S)=0$, otherwise. Applying the Shapley value to this restricted game gives the Myerson value of the communication graph game, giving payoffs $\operatorname{Sh}\left(v^{\mathcal{F}}\right)=\left(\frac{3}{10}, \frac{1}{20}, \frac{1}{20}, \frac{3}{10}, \frac{3}{10}\right)$.

Since an antimatroid $\mathcal{A}$ is union closed, any subset $S \subseteq N$ has a unique component given by the interior operator $\operatorname{int}(S)=\bigcup_{\{C \in \mathcal{A}: C \subseteq S\}} \bar{C}$, being the largest feasible 
subset of $S$, see Korte et al. [20]. In fact, the restricted game $v^{\mathcal{A}}: 2^{N} \rightarrow \mathbb{R}$ of Definition 4.1 boils down to the game $v^{\mathcal{A}}(S)=v(\operatorname{int}(S))$, see Algaba et al. ([14],[21]).

An allocation rule or value for games on accessible union stable network structures is a function $f: G A U S^{N} \rightarrow \mathbb{R}^{N}$, that assigns a payoff vector $f(v, \mathcal{F}) \in \mathbb{R}^{N}$ to every game in this class. The payoff vector $f(v, \mathcal{F}) \in \mathbb{R}^{N}$ gives an allocation of the worth, that can be earned in the game $v$ taking into account the relations in the network structure $\mathcal{F}$. We consider the allocation rule that assigns to every game on an accessible union stable network structure the Shapley value of the corresponding restricted game.

Definition 4.2 The allocation rule $\varphi: G A U S^{N} \rightarrow \mathbb{R}^{N}$ for games on accessible union stable network structures is defined by $\varphi(v, \mathcal{F})=\operatorname{Sh}\left(v^{\mathcal{F}}\right)$, for every $(v, \mathcal{F}) \in G A U S^{N}$, where $S h$ denotes the Shapley value, see (2).

For games on accessible union stable network structures, the allocation rule $\varphi$ generalizes the Myerson value for games restricted by communication graphs, and the (conjunctive and disjunctive) permission value for games with a permission structure.

Next, we provide an axiomatization of this Shapley-type value. Component efficiency of an allocation rule for games on accessible union stable network structures states that for every game in this class, the total payoff to every component equals its worth.

Definition 4.3 An allocation rule $f$ on $G A U S^{N}$ satisfies component efficiency, if $\sum_{i \in M} f_{i}(v, \mathcal{F})=v(M)$, for all $(v, \mathcal{F}) \in G A U S^{N}$ and $M \in C_{\mathcal{F}}(N)$.

A player $i \in N$ is called component dummy in an accessible union stable network structure $\mathcal{F}$, if this player does not belong to any feasible coalition, i.e. $i \notin S$, for every $S \in \mathcal{F}$. Note that a component dummy in $\mathcal{F}$ is a null player in any $v^{\mathcal{F}}$, that is derived from some $v \in \mathcal{G}^{N}$.

Definition 4.4 An allocation rule $f$ on $G A U S^{N}$ satisfies component dummy, if $f_{i}(v, \mathcal{F})=0$, for every component dummy $i$ in $\mathcal{F},(v, \mathcal{F}) \in G A U S^{N}$.

Inspired by the balanced contributions property of Myerson [22], we will compare the effect of deleting players from the accessible union stable network structure on each others payoff. Given a network structure $\mathcal{F} \subseteq 2^{N}$ and a player $i \in N$, the network structure

$$
\mathcal{F}_{-i}=\{S \in \mathcal{F}: i \notin S\}
$$

is given by all those feasible coalitions in $\mathcal{F}$ which do not contain player $i$. In order to compare payoffs, when deleting coalitions containing a particular player, we need to verify if the remaining collection of coalitions is still an accessible union stable network structure. This turns out to be the case.

Proposition 4.1 If $\mathcal{F} \subseteq 2^{N}$ is an accessible union stable network structure and $i \in N$, then $\mathcal{F}_{-i}$ is an accessible union stable network structure. 
Proof If $S, T \in \mathcal{F}_{-i}$, with $S \cap T \neq \emptyset$, then by union stability $S \cup T \in \mathcal{F}$. Since $i \notin S \cup T$, it holds that $S \cup T \in \mathcal{F}_{-i}$.

Moreover, if $S \in \mathcal{F}_{-i}$, then $S \in \mathcal{F}$, and applying accessibility there exists a $j \in S$ such that $S \backslash\{j\} \in \mathcal{F}$. Since $i \notin S$, it holds that $S \backslash\{j\} \in \mathcal{F}_{-i}$.

Observe that, if $\mathcal{F} \subseteq 2^{N}$ is an accessible union stable network structure and $i \in N$, then $i$ is a component dummy for the accessible union stable network structure $\mathcal{F}_{-i}$. By Proposition 4.1, we can define the following balanced contributions axiom, stating that deleting all coalitions containing a particular player $i$ has the same effect on the payoff of another player $j \neq i$, as the effect of deleting all coalitions containing $j$ has on the payoff of $i$.

Definition 4.5 An allocation rule $f$ on $G A U S^{N}$ satisfies balanced contributions, if for every $(v, \mathcal{F}) \in G A U S^{N}$ and any two players $i, j \in N$, with $i \neq j$, we have

$$
f_{i}(v, \mathcal{F})-f_{i}\left(v, \mathcal{F}_{-j}\right)=f_{j}(v, \mathcal{F})-f_{j}\left(v, \mathcal{F}_{-i}\right)
$$

Proposition 4.2 For all $(v, \mathcal{F}) \in G A U S^{N}$ and all $i, j \in N$, with $i \neq j$, the Shapley value satisfies balanced contributions.

Proof It is well-known that the Shapley value can be written, for every $v \in \mathcal{G}^{N}$, as

$$
S h_{i}(v)=\sum_{\{T \in \mathcal{F}: i \in T\}} \frac{\Delta_{v}(T)}{|T|} \text { for all } i \in N
$$

where $\Delta_{v}(T)$ is the Harsanyi dividend of coalition $\emptyset \neq T \subseteq N$, see Harsanyi [23]. ${ }^{4}$

Consider $i \in N$ and $j \in N \backslash\{i\}$. If $T \in \mathcal{F}_{-i}$, then every $S \in \mathcal{F}$ such that $S \subseteq T$ satisfies $i \notin S$, and hence, $S \in \mathcal{F}_{-i}$ and $S \backslash\{i\}=S$. Since $C_{\mathcal{F}_{-i}}(S)=C_{\mathcal{F}}(S \backslash\{i\})$, for all $S \subseteq N$, for $T \in \mathcal{F}_{-i}$, we have

$$
\begin{aligned}
\Delta_{v^{\mathcal{F}_{-i}}}(T) & =\sum_{S \subseteq T}(-1)^{|T|-|S|} v^{\mathcal{F}_{-i}}(S)=\sum_{S \subseteq T}(-1)^{|T|-|S|} v^{\mathcal{F}}(S \backslash\{i\}) \\
& =\sum_{S \subseteq T}(-1)^{|T|-|S|} v^{\mathcal{F}}(S)=\Delta_{v} \mathcal{F}(T)
\end{aligned}
$$

\footnotetext{
$\overline{{ }^{4} \text { For each } T \subseteq N \text {, the unanimity game }\left(N, u_{T}\right) \text { is given by } u_{T}(S)}=1$, if $T \subseteq S$, and $u_{T}(S)=0$, otherwise. It is well-known that the unanimity games $u_{T}, T \subseteq N, T \neq \emptyset$, form a basis of the vectorial space of TU-games on $N$. Each game $v \in \mathcal{G}^{N}$ can be written as a linear combination of unanimity games in a unique way as $v=\sum_{T \subseteq N}, T \neq \emptyset \Delta_{v}(T) u_{T}$. By applying the Möbius transformation, we obtain that $\Delta_{v}(S)=\sum_{T \subseteq S}(-1)^{|S|-|T|} v(T), \quad S \subseteq N$.
} 
Therefore, it holds that

$$
\begin{aligned}
\varphi_{i}(v, \mathcal{F})-\varphi_{i}\left(v, \mathcal{F}_{-j}\right) & =S_{i}\left(v^{\mathcal{F}}\right)-S_{i}\left(v^{\mathcal{F}_{-j}}\right) \\
& =\sum_{\{T \in \mathcal{F}: i \in T\}} \frac{\Delta_{v} \mathcal{F}(T)}{|T|}-\sum_{\left\{T \in \mathcal{F}_{-j}: i \in T\right\}} \frac{\Delta_{v} \mathcal{F}_{-j}(T)}{|T|} \\
= & \sum_{\{T \in \mathcal{F}: i, j \in T\}} \frac{\Delta_{v} \mathcal{F}(T)}{|T|} \\
= & \sum_{\{T \in \mathcal{F}: j \in T\}} \frac{\Delta_{v} \mathcal{F}(T)}{|T|}-\sum_{\left\{T \in \mathcal{F}_{-i}: j \in T\right\}} \frac{\Delta_{v^{\mathcal{F}}}(T)}{|T|} \\
= & S_{j}\left(v^{\mathcal{F}}\right)-S_{j}\left(v^{\mathcal{F}_{-i}}\right)=\varphi_{j}(v, \mathcal{F})-\varphi_{j}\left(v, \mathcal{F}_{-i}\right),
\end{aligned}
$$

where the third equality follows from $\Delta_{v} \mathcal{F}(T)=\Delta_{v} \mathcal{F}_{-j}(T), T \in \mathcal{F}_{-j}$.

The following theorem provides a characterization of the Shapley value on the class of games on accessible union stable network structures.

Theorem 4.1 The Shapley value is the unique allocation rule on GAUS ${ }^{N}$, that satisfies component efficiency, component dummy, and balanced contributions.

Proof Algaba et al. [3] show that the Shapley value satisfies component efficiency and component dummy on the class of games on union stable network structures and, therefore, also on $G A U S^{N}$. By Proposition 4.2, the Shapley value satisfies balanced contributions. Therefore, we only need to show uniqueness. Consider an allocation rule $f$ on $G A U S^{N}$ satisfying component efficiency, component dummy and balanced contributions. We will prove that $f(v, \mathcal{F})=\varphi(v, \mathcal{F})$, for all $(v, \mathcal{F}) \in G A U S^{N}$, by induction on the number of feasible coalitions.

If $|\mathcal{F}|=1$, then $\mathcal{F}=\{\emptyset\}$ and hence, every player $i \in N$ is a component dummy. By the component dummy property, $f_{i}(v, \mathcal{F})=0=\varphi_{i}(v, \mathcal{F}), i \in N$.

Let $k>1$ and suppose that $f(v, \mathcal{F})=\varphi(v, \mathcal{F})$ for all $(v, \mathcal{F}) \in \operatorname{GAUS}^{N}$ such that $|\mathcal{F}| \leq k-1$. Let $(v, \mathcal{F}) \in G A U S^{N}$ with $|\mathcal{F}|=k$. Observe that the grand coalition $N$ is the union of the set of component dummy players and the components of $N$. Therefore, it suffices to check that $f_{i}(v, \mathcal{F})=\varphi_{i}(v, \mathcal{F})$ for all $i \in M$ and $M \in C_{\mathcal{F}}(N)$. Let $M \in C_{\mathcal{F}}(N)$. If $|M|=1$, then component efficiency determines the payoff for $i \in M$. If $|M|>1$, take $i \in M$. For every $j \in M \backslash\{i\}$, repeatedly applying balanced contributions yields

$$
f_{i}(v, \mathcal{F})-f_{i}\left(v, \mathcal{F}_{-j}\right)=f_{j}(v, \mathcal{F})-f_{j}\left(v, \mathcal{F}_{-i}\right), \quad \text { for all } j \in M \backslash\{i\}
$$

This yields $|M|-1$ linear independent equations. Component efficiency requires

$$
\sum_{i \in M} f_{i}(v, \mathcal{F})=v(M)
$$


Since the values $f\left(v, \mathcal{F}_{-i}\right)$ and $f\left(v, \mathcal{F}_{-j}\right)$ are known by the induction hypothesis, (4) together with Eq. (3) yields $|M|$ linear independent equations in the $|M|$ unknown payoffs $f_{h}(\mathcal{F}, v), h \in M$, which are therefore uniquely determined. ${ }^{5}$

\section{Perspectives and Open Problems}

One of the open problems, regarding the application of accessible union stable network structures to cooperative games, is to consider effects on payoff allocation from deleting more than one player from the structure. Using balanced contributions, in the previous section, we considered the effects of deleting all coalitions, containing one particular player from the set of feasible coalitions on the payoffs of another player. Comparing payoffs of two players in this way, we can also consider the effect on their payoffs, when we delete all coalitions containing both players. So, for an accessible union stable network structure $\mathcal{F}$ and two players $i, j \in N$, we might consider the network structure $\mathcal{F}_{-i j}=\{S \in \mathcal{F}:\{i, j\} \nsubseteq S\}$, given by the collection of feasible coalitions in $\mathcal{F}$, that do not contain both players $i$ and $j$. In the literature, there are several 'fairness' axioms stating that the payoffs of different players change the same, if the network structure changes, in some sense, similar for these players. For accessible union stable network structures, an obvious property would be to equalize the changes in payoffs for players $i$ and $j$, when going from network structure $\mathcal{F}$ to network structure $\mathcal{F}_{-i j}$. A problem that arises is that, although $\mathcal{F}_{-i j}$ is also accessible, ${ }^{6}$ it may not be union stable. ${ }^{7}$ Therefore, we cannot simply apply this property to accessible union stable network structures.

Other versions of fairness are, for example, the extension of Myerson's [1] fairness to games on union stable network structures, as introduced in Algaba et al. [3], namely that for every support $B \in B(\mathcal{F})$, it holds that deleting $B$ from the basis of any union stable network structure, and extending the new (reduced) basis $B(\mathcal{F}) \backslash\{B\}$, by applying the union stability operator repeatedly untill the structure is union stable, the change in payoff of every player in $B$ is the same. In this case, the new network structure is union stable by construction, but may not be accessible. ${ }^{8}$ Exploring the possibilities to apply (modified) fairness axioms, to games on accessible union stable network structures, is a plan for future research.

\footnotetext{
5 Note that from this proof, it follows that this theorem holds for all classes of network structures, such that $\mathcal{F}_{-i}$ is in the class, for all $i \in N$. Although this follows from a more general result in Katsev [24], we provided the proof for completeness and to get a better insight in the structural properties.

6 To prove it, let $\mathcal{F} \subseteq 2^{N}$ be an accessible network structure. If $S \in \mathcal{F}_{-i j}$, then $S \in \mathcal{F}$, and thus, there is an $h \in S$ such that $S \backslash\{h\} \in \mathcal{F}$. Since $\{i, j\} \nsubseteq S$, it holds that $S \backslash\{h\} \in \mathcal{F}_{-i j}$. Hence, $\mathcal{F}_{-i j}$ is accessible.

7 Consider the accessible union stable network structure $\mathcal{F}$ of Example 2.1. Take a player from $N$ and one from $M$, for example, players 2 and 4 . Then, the network structure, defined as $\mathcal{F}_{-24}=$ $\mathcal{F} \backslash\{\{2,3,4,5\},\{1,2,3,4,5\}\}$, is not union stable, since $\{1,2\}$ and $\{1,3,4,5\}$ both belong to $\mathcal{F}_{-24}$, their intersection is nonempty, but their union does not belong to $\mathcal{F}_{-24}$.

8 Consider, for example, network structure of Example 3.2. Deleting support $\{3,4\}$ from the basis, and extending the reduced basis, we obtain $\mathcal{F} \backslash\{\{3,4\}\}$, which is not accessible, since $\{2,3,4\}$ does not have an extreme player anymore.
} 
Fig. 7 Two connected communication graphs on $N=\{1,2,3\}$

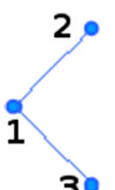

30

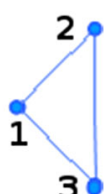

Further research will also include to consider the extension of the characteristic function, as in the network formation and stability model of Jackson and Wolinsky [25]. In their model, worths are not assigned to coalitions of players, but to communication network structures. They consider network value functions $\bar{v}: 2^{\mathcal{L}^{N}} \rightarrow \mathbb{R}$, with $\mathcal{L}^{N}=$ $\{\{i, j\}: i, j \in N, i \neq j\}$, being the collection of all undirected networks (i.e. sets of links) on player set $N$. For example, in the network structures $L=\{\{1,2\},\{1,3\}\}$, respectively, $L^{\prime}=L \cup\{\{2,3\}\}$ on $N=\{1,2,3\}$ (see Fig. 7) the 'grand coalition' $N$ can have a different value, although in a restricted game, in the sense of Myerson [1] (as considered in Sect. 4), coalition $N$ should have the same worth in both restricted games, because only connectedness matters. Since, in this paper, we studied more rich network structures than the bilateral communication networks, it is worthwhile to consider network value functions, similar to those in Jackson and Wolinsky [25], but for accessible union stable network structures and other structures.

\section{Conclusions}

In this paper, we introduced a new type of network structure, called accessible union stable network structure, which generalizes several well-known network structures, representing hierarchies or communication networks.

We showed that this new network structure contains the classes of undirected networks, antimatroids (hierarchies), as well as augmenting systems, and showed by an example of societies of explorers and careful players, that it is relevant to consider these general network structures.

We applied the new network structures to models of restricted cooperation in cooperative games and provided an axiomatization of the Shapley value using component efficiency, component dummy and balanced contributions. Whereas this generalization of balanced contributions, considering the deletion of a single player, goes smooth, the deletion of two (or more) players from these network structures is quite more complex, since the deletion of all feasible coalitions, containing two specific players, may violate union stability, while deleting a support from the network structure may not satisfy accessibility.

Acknowledgements This research is financially supported by the Netherlands Organization for Scientific Research, NWO Grant 400-08-026 and the Spanish Projects ECO2015-68856-P and MTM2014-54199-P. The stay of Encarnación Algaba in the Department of Econometrics and Tinbergen Institute was financially supported by VPPI-US. We thank Gerard van der Laan for valuable comments.

Open Access This article is distributed under the terms of the Creative Commons Attribution 4.0 International License (http://creativecommons.org/licenses/by/4.0/), which permits unrestricted use, distribution, 
and reproduction in any medium, provided you give appropriate credit to the original author(s) and the source, provide a link to the Creative Commons license, and indicate if changes were made.

\section{References}

1. Myerson, R.B.: Graphs and cooperation in games. Math. Oper. Res. 2, 225-229 (1977)

2. Algaba, E., Bilbao, J.M., Borm, P., López, J.: The position value for union stable systems. Math. Methods Oper. Res. 52, 221-236 (2000)

3. Algaba, E., Bilbao, J.M., Borm, P., López, J.: The Myerson value for union stable structures. Math. Methods Oper. Res. 54, 359-371 (2001)

4. Algaba, E., Bilbao, J.M., van den Brink, R., López, J.: The Myerson value and superfluous supports in union stable systems. J. Optim. Theory Appl. 155, 650-668 (2012)

5. Algaba, E., Bilbao, J.M., van den Brink, R.: Harsanyi power solutions for games on union stable systems. Ann. Oper. Res. 225, 27-44 (2015)

6. Algaba, E., Bilbao, J.M., López, J.: The position value in communication structures. Math. Methods Oper. Res. 59, 465-477 (2004)

7. Algaba, E., Bilbao, J.M., López, J.: A unified approach to restricted games. Theory Decis. 50, 333-345 (2001)

8. Gilles, R.P., Owen, G., van den Brink, R.: Games with permission structures: the conjunctive approach. Int. J. Game Theory 20, 277-293 (1992)

9. Gilles, R.P., Owen, G.: Cooperative Games and Disjunctive Permission Structures. Department of Economics, Virginia Polytechnic Institute and State University, Blacksburg (1994)

10. van den Brink, R., Gilles, R.P.: Axiomatizations of the conjunctive permission value for games with permission structures. Games Econ. Behav. 12, 113-126 (1996)

11. van den Brink, R.: An axiomatization of the disjunctive permission value for games with a permission structure. Int. J. Game Theory 26, 27-43 (1997)

12. Faigle, U., Kern, W.: The Shapley value for cooperative games under precedence constraints. Int. J. Game Theory 21, 249-266 (1992)

13. Algaba, E., van den Brink, R., Dietz, C.: Power measures and solutions for games under precedence constraints. J. Optim. Theory Appl. 172, 1008-1022 (2017)

14. Algaba, E., Bilbao, M., van den Brink, R., Jiménez-Losada, A.: Cooperative games on antimatroids. Math. Methods Oper. Res. 282, 1-15 (2004)

15. Dilworth, R.P.: Lattices with unique irreducible decompositions. Ann. Math. 41, 771-777 (1940)

16. Edelman, P.H., Jamison, R.E.: The theory of convex geometries. Geom. Dedic. 19, 247-270 (1985)

17. van den Brink, R.: On hierarchies and communication. Soc. Choice Welf. 39, 721-735 (2012a)

18. Bilbao, J.M.: Cooperative games under augmenting systems. SIAM J. Discrete Math. 17, 122-133 (2003)

19. Shapley, L.S.: A value for $n$-person games. In: Kuhn, H.W., Tucker, A.W. (eds.) Contributions to the Theory of Games, vol. 2, pp. 307-317. Princeton University Press, Princeton (1953)

20. Korte, B., Lovász, L., Schrader, R.: Greedoids. Springer, Berlin (1991)

21. Algaba, E., Bilbao, M., van den Brink, R., Jiménez-Losada, A.: Axiomatizations of the Shapley value for games on antimatroids. Math. Methods Oper. Res. 57, 49-65 (2003)

22. Myerson, R.B.: Conference structures and fair allocation rules. Int. J. Game Theory 9, 169-182 (1980)

23. Harsanyi, J.C.: A bargaining model for cooperative $n$-person games. In: Tucker, A.W., Luce, R.D. (eds.) Contributions to the Theory of Games, vol. IV, pp. 325-355. Princeton University Press, Princeton (1959)

24. Katsev, I.: The Shapley value for games with restricted cooperation. Working Paper, St. Petersburg Institute for Economics and Mathematics (2013)

25. Jackson, M.O., Wolinsky, A.: A strategic model of social and economic networks. J. Econ. Theory 71, 44-74 (1996) 